night shift work, frequency of night duties, total duration of rotating night shift work and lifestyle factors, i.e. a) smoking cigarettes, b) alcohol consumption, c) physical activity and d) BMI were examined with logistic regression and linear regression analyses adjusted for age.

Results Smoking cigarettes was associated significantly with current rotating night shift work $(\mathrm{OR}=1.4)$, frequency of night shifts $(\mathrm{OR}=1.5$ and $\mathrm{OR}=1.7$ among women with $5-7$ and $\geq 8$ night duties/month, respectively) and longer duration of the night shift work $(\mathrm{OR}=2.1$ for duration $>25 \mathrm{yrs})$. The total physical activity was higher among rotating night shift nurses (242 vs. 203 MET*h/week), but OR of recreational inactivity was significantly increased among rotating night shift workers $(\mathrm{OR}=1.6)$. Mean BMI was significantly higher among postmenopausal women working night shifts when compared to day workers $\left(\mathrm{BMI}=28.9\right.$ vs. $\left.27.6 \mathrm{~kg} / \mathrm{m}^{2}\right)$, with increased OR of obesity $(\mathrm{OR}=2.8)$. No significant associations were observed between night shift work and alcohol consumption.

Conclusions The results of our study indicate that rotating night shift work may be associated with poorer lifestyle, which may contribute to chronic diseases.

\section{LEVELS OF IDEAL, INTERMEDIATE AND POOR CARDIOVASCULAR HEALTH BY EMPLOYMENT CHARACTERISTICS IN 2005-06 NATIONAL HEALTH AND NUTRITION EXAMINATION SURVEY}

Tara Hartley, Cecil Burchfiel, Ja Gu, Michael Andrew. National Institute for Occupational Safety and Health, Morgantown, WV, USA

\subsection{6/oemed-2014-102362.208}

Objectives Employed persons are considered healthier than the general population. Yet, between $5-18 \%$ of all coronary heart disease deaths can be attributed to occupational exposures, ranging from noise to job stress. Cardiovascular health $(\mathrm{CVH})$ is based on seven modifiable characteristics (i.e. cigarette smoking, body mass index (BMI), physical activity, diet, blood pressure, and levels of total cholesterol and fasting glucose) used to categorise individuals as having poor, intermediate and ideal CVH. In this study, we compared levels of $\mathrm{CVH}$ among employed and unemployed participants in the 2005-2006 National Health and Nutrition Examination Survey (NHANES).

Method The study population included 935 cardiovascular disease-free participants age 20 and older (731 employed, 204 unemployed). Employment status and work characteristics were derived from self-report questionnaires. Poor, intermediate and ideal levels of $\mathrm{CVH}$ and its components were defined using American Heart Association criteria. Weighted means and percentages were calculated using SUDAAN 10.0; models were adjusted for age, sex and ethnicity.

Results Mean number of ideal CVH components was significantly higher for employed compared to unemployed participants; $17.7 \%$ of those employed had ideal $\mathrm{CVH}$ compared to $12.2 \%$ of those unemployed. Ideal $\mathrm{CVH}$ was significantly lower for those in construction, manufacturing and transportation industries (8.6\%) and in precision, product and transportation occupations (4.4\%). Ideal CVH was significantly higher for afternoon shift $(26.7 \%)$ compared to night/rotating (10.9\%) and day $(18.2 \%)$ shift workers.

Conclusions Understanding unique stressors and exposures for persons working in manufacturing, transportation and construction industries would be an important next step in designing interventions to improve their CVH.

\section{5}

ASSOCIATIONS OF SYMPTOMS OF DEPRESSION AND POSTTRAUMATIC STRESS DISORDER WITH PERITRAUMATIC DISSOCIATION, AND THE ROLE OF TRAUMA PRIOR TO POLICE WORK

${ }^{1}$ Erin McCanlies, ${ }^{1}$ Khachatur Sarkisian, 'Michael Andrew, ${ }^{1}$ Cecil Burchfiel, ${ }^{2}$ John Violanti. ${ }^{1}$ National Institute for Occupational Safety and Health, Morgantown, WV, USA; ${ }^{2}$ University at Buffalo, State University of New York, Buffalo, NY, USA

10.1136/oemed-2014-102362.209

Objectives Our objective was to determine if symptoms of depression and posttraumatic stress disorder (PTSD) are associated with peritraumatic dissociation, and if this association is modified by trauma prior to police work.

Method Symptoms of depression, PTSD symptoms, peritraumatic dissociative experience (PDE), and trauma before police work were measured using the Centre for Epidemiologic Studies Depression scale, PTSD Check List-Civilian Version, PDE questionnaire, and the brief trauma questionnaire, respectively in 328 police officers. Separate regression models were used to assess if either symptoms of depression or symptoms of PTSD were associated with PDE stratified by prior trauma. Means were adjusted for race, number of drinks per week, and smoking.

Results PDE was significantly positively associated with symptoms of PTSD and depression $(\beta=0.642, \mathrm{p}=0.0001$ and $\beta=$ $0.276, \mathrm{p}=0.0002$, respectively). PDE was positively associated with symptoms of PTSD regardless of trauma before police work $(\beta=0.599, \mathrm{p}<0.0001$ (without prior trauma), $0.750, \mathrm{p}<$ 0.0001 (with prior trauma). In contrast to PTSD, depression symptoms were significantly associated with PDE scores in individuals with prior trauma $(\beta=0.466, \mathrm{p}=0.0001)$, but not in individuals without prior trauma $(\beta=0.130, \mathrm{p}=0.155)$.

Conclusions The results indicate that an increase in PDE is associated with an increase in symptoms of depression and PTSD. The results also show that PDE is associated with symptoms of PTSD regardless of prior trauma. In contrast, PDE was associated with depression symptoms only in individuals with prior trauma, indicating prior trauma may modify this relationship.

\section{PSYCHOSOCIAL WORK FACTORS, OCCUPATIONAL NOISE EXPOSURE, COMMON MENTAL DISORDERS, AND THE RISK OF TINNITUS}

1,2Thomas Winther Frederiksen, ${ }^{3}$ Cecilia H Ramlau-Hansen, 'Zara A Stokholm, ${ }^{1}$ Jesper M Vestergaard, ${ }^{1}$ Henrik A Kolstad. ${ }^{1}$ Department of Occupational Medicine, Danish Ramazzini Centre, Aarhus University Hospital, Aarhus, Denmark; ${ }^{2}$ Department of Otology, Head and Neck Surgery, Hospitalsenheden Vest, Holstebro, Denmark; ${ }^{3}$ Department of Public Health, Section for Epidemiology, Aarhus University, Aarhus, Denmark

\subsection{6/oemed-2014-102362.210}

Objectives Tinnitus is common, can be disabling, and may impair concentration, hearing and sleep. Noise induced hearing loss, other subtypes of hearing loss and ototoxic drugs are well-documented risk factors for tinnitus. Psychosocial work factors, depression and anxiety may exacerbate tinnitus, cause tinnitus, or both. The objective is to investigate the relationship between noise exposure, psychosocial work factors, common mental disorders, and tinnitus

Method A total of 554 workers within 10 manufacturing trades and children day-care participated in this cross-sectional study from 2009-2010. The study database contained information on individual short-term and long-term noise exposure levels, hearing levels and questionnaire information on common mental disorders and psychosocial work factors. Associations between 
noise exposure levels, hearing levels, depression, anxiety, burnout symptoms, work-related stress and tinnitus will be analysed by use of multiple logistic regression analysis, taking a priori selected potential confounders into account.

Results Preliminary results show that of the 554 participants, $77 \%$ were males an the mean age was 43 years, ranging from 20-64 years. Among the participants, 17\% reported tinnitus, $16 \%$ had a hearing handicap (WHO-definition), 3\% anxiety disorder, 14\% burn-out symptoms, $4 \%$ depression, and $8 \%$ reported work-related stress.

Conclusions Tinnitus is expected to represent a heterogeneous group of underlying disorders. We aim at contributing to a better understanding of the relative importance of the possible underlying factors in a population of occupationally noise exposed workers. Risk analyses are in progress, and results will be presented at the conference.

\section{THE TREND IN THE PREVALENCE OF CHILDHOOD HEARING IMPAIRMENT IN TAIWAN AND ITS IMPLICATIONS}

\begin{abstract}
1,2 2 Der-Chung Lai, ${ }^{3}$ Yen-Cheng Tseng, ${ }^{4,5}$ Cheng-Yu Lin, ${ }^{5,6}$ How-Ran Guo. ${ }^{1}$ Ditmanson Medical Foundation Chia-Yi Christian Hospital, Chia-Yi, Taiwan; ${ }^{2}$ Chia Nan University of Pharmacy and Science, Tainan, Taiwan; ${ }^{3}$ Chang Jung Christian University, Tainan, Taiwan; ${ }^{4}$ Tainan Hospital, Ministry of Health and Welfare, Tainan, Taiwan; ${ }^{5}$ National Cheng Kung University Hospital, Tainan, Taiwan; ${ }^{6}$ National Cheng Kung University, Tainan, Taiwan
\end{abstract}

\subsection{6/oemed-2014-102362.211}

Objectives Childhood hearing impairment (CHI) is a major developmental disability, but data at the national level are limited, especially those on the trends over time. We conducted a study to assess the time trend of CHI prevalence in Taiwan and explore its associated factors.

Method The Taiwan government certifies disabled residents for providing various services and maintains a registry of certified cases. We analysed the registry data on cases under 17 years old from 2000 to 2011 to estimate the prevalence of CHI and assess the time trend.

Results Each year, registered cases ranged from 3427 to 4075 . The prevalence increased from 2000 to 2006, and then decreased till 2011. In general, the prevalence increased over the years in the age groups $<3$ years, 3-5 years, and 6-11 years $(p<0 \cdot 05)$, but decreased in the age group 12-14 years $(p<0.05)$. The largest increase was observed in the age group $<3$ years, particularly after the promotion of screening by the government in 2003. The decrease after 2006 was mainly attributable to decreases in the age groups $12-14$ and $15-17$ years, and similar decreases had been observed in countries with rubella vaccination programs.

Conclusions In Taiwan, the prevalence and proportion of $\mathrm{CHI}$ in the age group $<3$ years had increased from 2000 to 2006 after the implementation of hearing screening programs. The overall prevalence decreased from 2006 to 2011, which might be attributable to the improvements in medical care and the nationwide rubella vaccination program.

\section{A STRUCTURAL EQUATION MODELLING METHOD TO DESCRIBE WORK ENVIRONMENT RISK FACTORS AND MUSCULOSKELETAL SYMPTOMS AMONG HOSPITAL NURSES}

Alireza Dehdashti. Semnan University of Medical Sciences, Semnan, Iran
Objectives Epidemiological studies have shown that musculoskeletal symptoms are frequent occupational injury and disability among nurses in developing countries. Preventive measures were not able to reduce musculoskeletal disorders significantly in nursing profession. This study was performed to provide a comprehensive assessment of the association among risk factors in developing musculoskeletal symptoms.

Method A structural equation model was applied to describe and analyse complex causal relationships from sets of occupational variables involved in musculoskeletal symptoms. A questionnaire was applied to nurses at hospitals affiliated to Semnan Medical Sciences University to collect data on personal and occupational factors and musculoskeletal symptoms. An ergonomic rapid entire body assessment measured work posture risks.

Results Physical work demand and mental pressure increased the risk of musculoskeletal disorders significantly. Musculoskeletal symptoms increased in nurses who worked for extended hours and experienced frequent unstable work posture. Structural equation model showed that musculoskeletal symptoms were associated directly and indirectly by physical and mental job variables, employment status, age and lifestyle.

Conclusions Covarience structural analysis is useful to describe and understand both the direct and indirect effects of variables with complex relationship between risk factors on the prediction of musculoskeletal symptoms.

\section{PTSD AND COGNITIVE IMPAIRMENT AMONG RESCUE AND RECOVERY WORKERS EXPOSED TO THE 9/11 WORLD TRADE CENTRE DISASTER}

${ }^{1}$ Sadie Sanchez, ${ }^{1}$ Stephanie Barnhart, ${ }^{2}$ Steven Stellman, ${ }^{2}$ James Cone, ${ }^{2}$ Robert Brackbill. ${ }^{1}$ Icahn School of Medicine at Mount Sinai, New York, NY, USA; ${ }^{2}$ World Trade Center Health Registry (NYCDOHMH), New York, NY, USA

\subsection{6/oemed-2014-102362.213}

Objectives The 9/11 World Trade Centre Disaster (WTCD) has resulted in many chronic health problems among survivors. Rescue/recovery workers (RRW), having the highest exposures, would likely be at the highest risk for these problems. We sought to evaluate cognitive impairment among RRW with and without prevalent posttraumatic stress disorder (PTSD) which may act as a mediator.

Method The World Trade Centre Health Registry is a voluntary longitudinal cohort study. Surveys collected demographics as well as 9/11 related exposures and health outcomes. We limited analysis to RRW workers only. WTCD exposure among RRW was classified from low to very high based on a sum of twelve 9/ 11 exposures. Cognitive impairment was defined by the frequency of trouble remembering things over the prior 7 days, and presence and/or worsening of memory loss or confusion over the prior 12 month period. A PTSD checklist score of 44 or greater was used to assess the presence of probable PTSD.

Results Of 18932 RRW, 2436 (12.9\%) had PTSD while 16506 (87.1\%) did not have PTSD. Among those without PTSD, 7.9\% in the low exposure group were cognitively impaired, $8.9 \%$ with medium exposure, $10.3 \%$ with high exposure, and $14.4 \%$ with very high exposure. Among those with PTSD, $33.6 \%$ in the low exposure group were cognitively impaired, $33.5 \%$ with medium exposure, $32.5 \%$ with high exposure, and $38.1 \%$ with very high exposure.

Conclusions In WTCD RRW without PTSD, there is a doseresponse for cognitive impairment with increased exposure. 